\title{
Effects of hereditary moderate high fat diet on metabolic performance and physical endurance capacity in C57BL/6 offspring
}

\author{
FANG LIU $^{1}$, GUIRONG ZHANG ${ }^{1}$, XUE SHENG ${ }^{1}$, SHANG LIU $^{1}$, MENG CUI ${ }^{1}$, \\ HEYUN GUO $^{1}$, JIANBIN XUE ${ }^{1}$ and LINGYUN ZHANG ${ }^{2}$ \\ ${ }^{1}$ College of Life Science, School of Life Science, Jilin University, Changchun, Jilin 130012; \\ ${ }^{2}$ Department of Obstetrics and Gynecology, Zhu Jiang Hospital, Southern Medical University, \\ Guangzhou, Guangdong 510000, P.R. China
}

Received February 18, 2016; Accepted March 6, 2017

DOI: $10.3892 / \mathrm{mmr} .2018 .8432$

\begin{abstract}
Obesity in pregnant women presents a risk to fetal health, leading to numerous metabolic syndromes and chronic inflammation risks. Previously, physical exercise was considered to be one of the primary treatments for obesity. However, the effect of fat consumption throughout the life cycle on physical endurance capacity remains unknown. A total of two groups of female mice (age, 6 weeks; C57BL/6J) were fed with a normal chow diet and a moderate high fat diet (MHFD), during pregnancy and lactation (8 weeks), with the offspring receiving the same diet as the mother. When filial mice were 8,16 and 24 weeks old, they were tested for endurance, blood pressure (BP) and glucose tolerance, as well as adipose tissue infiltration and macrophage subtype. Compared with the control group, filial mice in MHFD groups exhibited increased BP and glucose levels and larger adipose cells ( 4 -fold). During adolescence, the obese filial mice demonstrated increased endurance compared with controls. Endurance declines in middle and old age; the endurance of aged obese mice was $29 \%$ that of lean ones. In addition, body coordination and movement memory did not notably change. The expression of cluster of differentiation 68 , one of the most reliable markers of macrophages, increased by 2.48 -fold, demonstrating that macrophages were recruited and underwent infiltration. In addition, increased tumor necrosis factor- $\alpha$ and decreased interleukin-10 expression demonstrated that
\end{abstract}

Correspondence to: Dr Lingyun Zhang, Department of Obstetrics and Gynecology, Zhu Jiang Hospital, Southern Medical University, 253 Industrial Street, Guangzhou, Guangdong 510000, P.R. China E-mail: zhangly7225@hotmail.com

Dr Guirong Zhang, College of Life Science, School of Life Science, Jilin University, 2699 Qianjin Street, Changchun, Jilin 130012, P.R. China

E-mail: zgr@jlu.edu.cn

Key words: offspring, moderate high fat diet, physical endurance, interleukin-10/tumor necrosis factor- $\alpha$ ratio infiltrated macrophages are polarized to the M1 state, which weakens physical endurance and resists type M2 macrophages, which exhibit repairing functions. In conclusion, hereditary MHFD weakens physical endurance and alters the metabolic characteristics of C57BL/6 offspring.

\section{Introduction}

Obesity is a medical disorder characterized by excessive fat accumulation that presents a risk to health, leading to reduced life expectancy and increased morbidity (1). These risks most commonly present in the following ways: Metabolic syndrome, hypertension, imprinting of metabolic control in fetal life and early childhood, and physical inactivity (1). Numerous studies have demonstrated the association between a sedentary lifestyle and weight gain; however, reliable direct measures of physical activity are only now beginning to emerge (1). High fat-fed wild-type (WT) mice demonstrated reduced exercise tolerance during an exercise stress test, and attenuation in muscle glucose uptake and AMP-activated protein kinase $\alpha-2$ activity during a single bout of exercise (2). However, recent work has identified that older adults with obesity and systemic inflammation have associated metabolic dysfunction (3), although they do not have associated reduced muscular weight or strength. In 2013, the American Medical Association classified obesity as a disease (3).

Obesity in pregnancy has become a global problem. In 2008, 64\% of American women of child-bearing age were overweight or obese (4). According to the Chinese adult Body Mass Index classification, $11.9 \%$ of pregnant females were overweight and $2.3 \%$ were obese at the first prenatal visit, increasing from 6.9-17.5 and 1.0-4.0\%, respectively between 2006 and 2009 (5). The traditional idea that pregnant women require compensatory nutrition will further drive the increase in obesity during pregnancy.

In 1992, Hales and Barker (6) published a hypothesis following research at the University of Southampton (Southampton, UK), which supported the idea that fetal development is modified in poor nutritional conditions, resulting in a thrift phenotype. It was concluded that infants whose 
birth weight fell on the low end of normal were more likely to die of heart disease as adults. Subsequently, Baker identified that the maternal environment impacts fetal development in ways which remain to be completely elucidated (7). In 2014, researchers at Yale University (New Haven, CT, USA) demonstrated that children of obese mothers who eat a high fat diet may be more likely to have metabolic disorders, and be at an increased risk of becoming obese and developing diabetes through hypothalamic neuro-circuit formation (8). Macrophage infiltration in the adipose tissue of obese animals, in response to free fatty acids released by hypertrophied adipocytes (9), contributes to inflammation and insulin resistance (10). Investigating the expression of transcripts encoding cluster of differentiation CD68, a macrophage transmembrane protein, has led to an improved understanding of the association between adipose tissue infiltration and insulin resistance (11). There is increasing evidence that an increase in the gene expression of tumor necrosis factor- $\alpha$ (TNF- $\alpha$ ) and interleukin-6 (IL-6) occurs in the hypothalamus in the offspring of obese mothers, considered to be produced by hypertrophied adipocytes as a marker of M1 polarization of adipose tissue macrophages $(9,10)$.

The controversy of alternative (M2) macrophage polarization has previously been debated. Although the majority of reports demonstrated a decline in M2 macrophages (10), researchers at Cornell University (Ithaca, NY, USA) observed elevated M2 macrophage polarization in adipose tissue with an acute high fat diet (HFD) challenge (12). An increase in IL-10 caused by the increase of M2 macrophages was reported in pregnant mice (13).

Skeletal muscle is required for movement and to sustain posture, and a loss of skeletal muscle occurs as consequence of several chronic diseases as well as normal aging (14). In addition, TNF- $\alpha$ expression and systemic inflammation was demonstrated to be associated with impaired angiogenesis in skeletal muscle through von Hippel-Lindau disease tumor suppressor overexpression (15).

In the present study, the effects of moderate HFD (MHFD) on the offspring of obese mice were investigated, including blood pressure (BP), physical inactivity, glucose sensitivity, macrophage infiltration, and the association between cytokine fluctuations and physical inactivity.

\section{Materials and methods}

Animal care. The female C57BL/6J mice and their controls ( $\mathrm{n}=64,6$-weeks-old, $\sim 17 \pm 2.2 \mathrm{~g}$ ) were purchased from the animal center of Norman Bethune College of Medicine, Jilin University (Changchun, China). All of the mice were maintained under a 12/12 h light-dark cycle at a constant temperature $\left(20 \pm 2^{\circ} \mathrm{C}\right)$ in the pathogen-free facilities at the Biological Experimental Teaching Demonstration Center of Jilin University, with food and water available ad libitum. The experimental animal protocol used in the present study was approved by the ethics committee of the School of Life Sciences at Jilin University.

Obesity mouse model. Following a 3-day acclimation period, C57BL/6J female mice at age 6 weeks were fed randomly with either normal chow diet (NCD), with $9 \%$ fat, or MHFD, with $26 \%$ fat. At 14 weeks of age, the female mice fed with NCD or MHFD were bred with C57BL/6J male mice $(n=8$, 6 -months-old, $\sim 18 \pm 1.7 \mathrm{~g}$ ). In order to improve the rate of successful mating, the male mice and the female mice were kept in one cage for 3 days. The female mice were examined every morning for the presence of a vaginal plug; the day following identification of a vaginal plug was designated day 0 of gestation. The maternal mice were housed individually with free access to their prior diet and water. Body weight and food intake were monitored weekly between weeks 6 and 14, and on days 0,7 and 14 of gestation. Female mice with $<7$ or $>10$ offspring were excluded from the present study, as described previously (9). Offspring were fed with NCD and MHFD separately, and separated from the maternal mice at the 4th week following birth.

Sampling. In the 8, 16 and 24th weeks, following an overnight fast, the mice were sacrificed by cervical dislocation for the collection of blood and tissue samples. When blood was visible in the eyes, the abdomen was rapidly opened and single samples of subcutaneous adipose tissue, parametrial or epididymal adipose tissue, perirenal adipose tissue and mesenteric adipose tissue were dissected and weighed to determine visceral fat content. The subcutaneous adipose tissue samples were either fixed with $4 \%$ formalin for $48 \mathrm{~h}$ at room temperature and embedded in paraffin, or frozen by dry ice-isopentane and optimal cutting temperature compound (OCT; Sakura Finetek USA, Inc., Torrance, CA, USA)-embedded for at $\sim 69^{\circ} \mathrm{C}$ for histological analyses. The heart, liver, spleen, lungs and kidneys were also rapidly removed, weighed, frozen in liquid nitrogen and stored at $-80^{\circ} \mathrm{C}$. The subtraction method was used to measure The wet weight of organs and fat tissues from the sacrificed mice were measured by the subtraction method: Total weight of containers and tissues minus the weight of the container.

Treadmill test. The exhaustion treadmill test was conducted to measure the physical performance of the skeletal muscle of the fetal mice. Prior to the exhaustion test, each mouse was placed on the belt of a six-lane motorized treadmill (FT-100 Animal Treadmill) at an incline of $0^{\circ}$ and speed of $12.28 \mathrm{~m} / \mathrm{min}$ for 5 min. As physical condition declined with age, the optimum speed for older mice was selected to analyze mice of all groups. Subsequent to three repeats for acclimation, the exhaustion test was carried out until mice stayed on the shaker plate for more than $10 \mathrm{sec}$ without attempting to run. The exhaustion time and distance were recorded for each subject $(16,17)$.

Rotarod test. In order to assess motor coordination and motor learning, the fetal mice were trained three times on the rotarod (ZB-200 Rotarod; Timen Co.), as described by Jung et al $(18,19)$. The apparatus consisted of a polyvinyl chloride rotating rod with 6 opaque Plexiglas barriers dividing the rod into sections, which exhibited individual holding chambers located $39 \mathrm{~cm}$ below the rod. Each mouse was put on the rod individually, facing away from the experimenter, and the rod was programmed to accelerate to $16 \mathrm{rpm}$. The time at which the mice fell from the rod was recorded. Following $\geq 10 \mathrm{~min}$ rest, the mice were placed back on the rod. Each mouse was tested 3 times and the mean latency to fall, across the three trials, was analyzed. 
BP measurement. BP was measured by tail-cuff plethysmography, as described previously by $\mathrm{Xu}$ et al (20). The measurements were conducted in a heated room $\left(30^{\circ} \mathrm{C}\right)$ in order to get optimal BP readings, and at the same time of day. Once the animals were restrained properly, heart rate (HR), systolic $\mathrm{BP}(\mathrm{SBP})$, mean BP (MBP) and diastolic BP (DBP) were automatically measured by BP2010 (Softron Corp., Tokyo, Japan). A total of $\geq 5$ readings were taken from each animal/session and averaged to obtain a single session value. BP was measured at 8, 16 and 24 weeks post-weaning. At each time-point, the average BP values were taken from 6-12 offspring with equal numbers of each gender in each treatment group.

Glucose tolerance test. The glucose tolerance test was carried out on the pregnant mice at 14 weeks, and on the offspring at each time-point (8, 16 and 24 weeks). The mice fasted overnight and were administered a glucose solution ( $1 \mathrm{~g} / \mathrm{kg}$ body weight) by subcutaneous injection. Blood samples were collected from a tail vein, and the glucose concentration measurement was performed during the light phase and determined using a blood glucose meter (SanoCare, Inc., FL, USA) (9).

Histopathological examination. A section of offspring subcutaneous abdominal adipose tissue was fixed in $4 \%$ formalin in PBS for $48 \mathrm{~h}$ at room temperature and dehydrated in gradient ethanol (50, 70, 80, 90, 95 and 100\%). Samples were embedded in paraffin, and cut into serial sections at $5 \mu \mathrm{m}$ thickness using a microtome (Leica Microsystems GmbH, Wetzlar, Germany). The sections were stained with hematoxylin at room temperature for $10 \mathrm{~min}$ and images were captured using an upright microscope (magnification, x10 and x40; Eclipse ci; Nikon Corp., Tokyo, Japan). Further sections of offspring subcutaneous adipose tissue, embedded in OCT, were cut into $20 \mu \mathrm{m}$ sections and mounted on glass slides, and stained with Oil Red $\mathrm{O}$ at room temperature for $10 \mathrm{~min}$. The three fields of vision were selected at random. The diameter of each adipocyte in the field was measured manually, and the diameters of 20 adipocytes were measured microscopically by a single observer, as described previously (13).

RNA isolation and reverse transcription (RT). A total of 6 offspring were randomly selected from each litter for each of the 3 time-points. Total RNA isolation from subcutaneous adipose tissue was conducted by the Trizol method as described previously by Campbell with minor modification (20). RT of total RNA was carried out with the PrimeScript RT reagent kit with genomic DNA Eraser (cat. no. RR047A; Takara Biotechnology Co., Ltd., Dalian, China). DNA contamination was removed from the total RNA before its reverse transcription to cDNA according to the manufacturer's protocol.

Quantitative polymerase chain reaction ( $q P C R)$. qPCR was performed using TaqMan gene expression to measure the expression of cluster of differentiation (CD)68 in subcutaneous adipose tissue. TaqMan probes and primers were purchased from Takara Biotechnology Co., Ltd. as follows: Mouse CD68, forward, 5'-GCTACTAGTCCAAGATCC-3' and reverse, 5'-CCTGAATTGGGTATAGGA-3' and probe, 5'-(FAM)CAC TGTTGGCCCTCACCCTG(Eclipse)-3'; and mouse GAPDH, forward, 5'-CAATGTGTCCGTCGTGGATCT-3' and reverse,
5'-GTCCTCAGTGTAGCCCAAGATG-3' and probe, CGT GCCGCCTGGAGAAACCTGCC(Eclipse)-3'.

qPCR analysis was performed in a $20 \mu \mathrm{l}$ volume using Premix Ex Taq ${ }^{\mathrm{TM}}$ (cat. no. RR390A; Takara Biotechnology Co., Ltd.) and Applied Biosystems 7500 (Thermo Fisher Scientific, Inc., Waltham, MA, USA), and the three-step RT-qPCR was performed under the following conditions: Denaturation at $95^{\circ} \mathrm{C}$ for $30 \mathrm{sec}$ followed by 40 cycles of denaturing at $95^{\circ} \mathrm{C}$ for $5 \mathrm{sec}$, annealing at $55^{\circ} \mathrm{C}$ for $10 \mathrm{sec}$ and extension at $72^{\circ} \mathrm{C}$ for $30 \mathrm{sec}$. Each reaction was repeated three times. Quantification was conducted using the $2^{-\Delta \Delta \mathrm{Cq}}$ method (21).

Serum TNF- $\alpha$ and $I L-10$ protein levels. An inflammatory cytokine, TNF- $\alpha$, and an anti-inflammatory cytokine, IL-10, were detected in the plasma of the offspring using mouse TNF- $\alpha$ and mouse IL-10 ELISA (MTA00B and DY417-05, R\&D Systems, Inc., Minneapolis, MN, USA), which were conducted according to the manufacturers' protocol.

Statistical analyses. Results were expressed as the mean \pm standard error. Comparisons of body weight and food intake were made using the paired-samples t-test. Other multi-group comparisons of biochemical and biophysical parameters in the offspring and maternal mice, were made using independent-samples t-tests using SPSS software (version 19.0; IBM SPSS, Armonk, NY, USA). P<0.05 was considered to indicate a statistically significant difference.

\section{Results}

Maternal body weight and food intake, fetal body weight and prime body weight. Following 8 weeks of treatment with NCD or MHFD, the female MHFD mice were significantly heavier compared with the female NCD mice with an increase in body weight of $38.45 \%(\mathrm{P}<0.05)$, which gave rise to a continued weight gain (Fig. 1A) and increased food intake (Fig. 1B) following pregnancy. In the MHFD offspring group, body weight increased following the offspring being separated from the maternal mice, and plateaued at the age of 12 weeks. The offspring of MHFD mice were heavier compared with those of NCD mice throughout the experimental period (Fig. 1C). Correspondingly, a marked increase in prime body fat was observed in the MHFD offspring group at 8 and 24 weeks old (Fig. 1D).

Treadmill test and rotarod test. In the treadmill test, 8-week old MHFD mice with larger mass exhibited an extended running time until exhaustion of 47\%; at the age of 16 weeks, the endurance capacity of MHFD mice decreased by $37 \%$, and there was a $28 \%$ decline compared with the control group. The two groups of 24-week old mice underachieved; the MHFD mice ran $29 \%$ of the running distance of the NCD mice (Fig. 2A). Despite a difference in the performance of the rotarod test between the two 8-week old groups, no significant differences were observed between the MHFD and NCD mice of any age group (Fig. 2B).

Maternal and fetal BP. SBP was elevated among the 14-week old MHFD maternal mice compared with their controls (Table I). A trend was exhibited in the offspring of the 

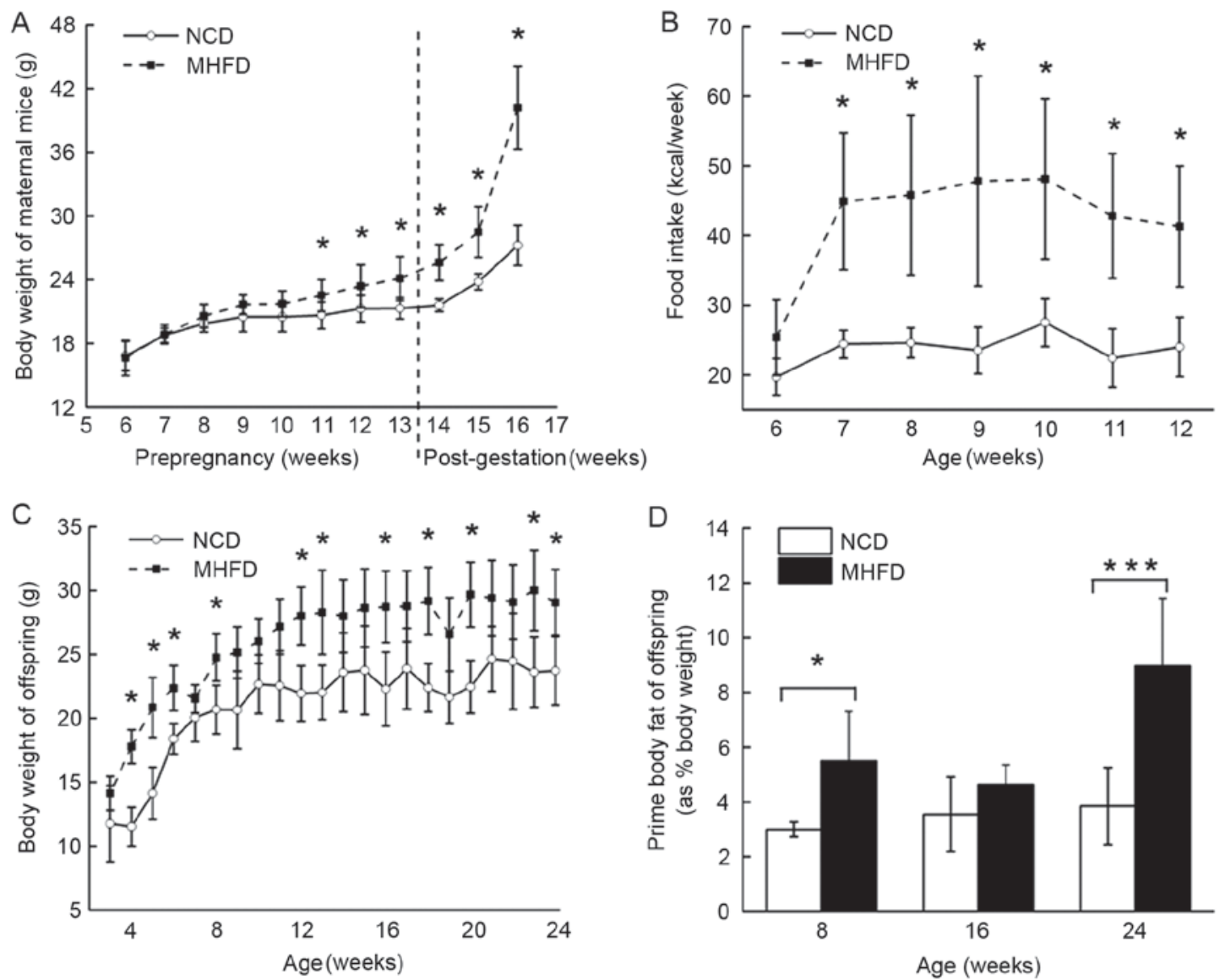

Figure 1. Comparisons of maternal and offspring body weights, maternal food intake and prime body fat. (A) Alterations in maternal body weight between 6 and 17 weeks of age, including the start of pregnancy between 14 weeks and late pregnancy (17 weeks) in NCD and MHFD groups (n=8). (B) Alterations in weekly maternal food calorie intake between 6 and 12 weeks of age in NCD and MHFD groups $(n=8)$. (C) Alterations in filial body weight between 3 and 24 weeks of age $(n=14)$. (D) Comparisons of filial prime body fat of the three age groups, containing single subcutaneous adipose tissue, single parametrial or epididymal adipose tissue, single perirenal adipose tissue and mesenteric adipose tissue between NCD and MHFD groups (n=8). Values are given as the mean \pm the standard error. $\mathrm{P}<0.05$ and ${ }^{* * *} \mathrm{P}<0.001$ vs. control. MHFD, moderate high fat diet; $\mathrm{NCD}$, normal chow diet.
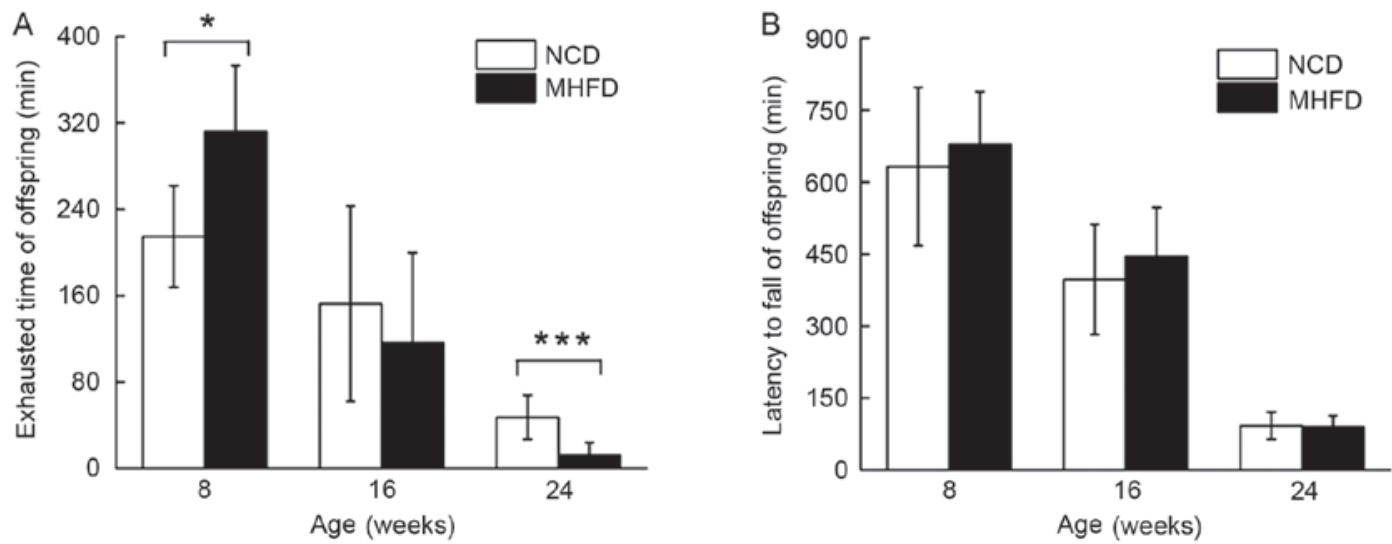

Figure 2. Alterations in physical endurance capacity and body coordination between offspring fed with NCD and those fed with MHFD. (A) Comparisons of exhaustion time in the treadmill test among the three age groups, between NCD and MHFD groups ( $\mathrm{n}=8$ ). (B) Latency to fall of the three age groups, between NCD and MHFD groups ( $\mathrm{n}=8)$. Values are given as the mean \pm the standard error. $\mathrm{P}<0.05$ and ${ }^{* * * *} \mathrm{P}<0.001$. MHFD, moderate high fat diet; NCD, normal chow diet.

MHFD and NCD groups, with increased BP observed among the MHFD offspring (Table I). It is notable that the group with the highest SBP measurements was not the oldest mice of 24 weeks, rather the 16-week groups (Table I). The data for MBP and DBP were consistent with that for SBP between the experimental groups (Table I). In addition, although the
MHFD mice exhibited an increase in HR in the maternal mice and the offspring, no significant difference between groups was observed (Table I).

Maternal and fetal glucose metabolism. The maternal mice were glucose intolerant (Fig. 3A). The glucose tolerance of 
the 8-week old MHFD offspring was comparable with that of the control group (Fig. 3B). A $14 \%$ and a $24 \%$ rise in the area under the curve of the glucose sensitivity measurements occurred in the 16-week and 24-week old MHFD offspring groups, respectively (Fig. 3C-E).

Major organs and issue of offspring. The subtraction method was used to measure the wet weight of organs and fat tissues from the sacrificed mice. As presented in Table II, the subcutaneous adipose tissue samples of the 8-week old MHFD offspring exhibited a $\sim 1.5$-fold increased mass compared with those of the 8-week old NCD offspring; this difference was increased between the two 16-week old groups, and ended with a 3-fold difference between 24-week old MHFD offspring and 24-week old NCD offspring. The kidneys of 24-week old MHFD mice also exhibited an increase in mass compared with the control group (Table II).

Morphological analysis of fetal adipose tissue. Consistent with previous studies, MHFD nutrition for 24 weeks caused significant adipocyte hypertrophy of $\sim 4$-fold, as demonstrated in paraffin sections stained with hematoxylin or frozen sections stained with Oil Red O (Fig. 4). The 24-week old MHFD mice exhibited fewer nuclei (Fig. 4A) and the cells were swollen with fat deposits (Fig. 4B), specifically stained using Oil Red O.

Inflammatory and anti-inflammatory alterations. Although no distinction was observed between the groups of young mice, the expression of transcripts encoding CD68 increased by 2.48-fold among the MHFD offspring compared with those of NCD mice (Fig. 5A). A 10\% decrease in serum IL-10 expression was exhibited among the young and the aged MHFD mice; however, the difference between the two young groups was not statistically significant (Fig. 5B). By contrast, the level of plasma TNF- $\alpha$ of the MHFD offspring was increased by $17 \%$ compared with the controls in the 8-week old groups, and peaked at $36.42 \%$ in the $24-w e e k$ old groups (Fig. 5C). The serum ratio of IL-10/ TNF- $\alpha$ among the NCD mice increased continuously, while remaining constant in the MHFD mice. Consequently, the IL-10/ TNF- $\alpha$ ratio was reduced in MHFD group compared with their controls throughout the experiment (Fig. 5D).

\section{Discussion}

The present study is, to the best of our knowledge, the first to investigate the physical endurance of obese C57BL/6 offspring throughout their life cycle. Obesity increases the likelihood of chronic diseases (1). Despite evidence that obesity is associated with hypertension, glucose sensitive, macrophage infiltration and adipokine changes $(1,22)$ these associations require further investigation.

As previously reported, BMI is markedly associated with SBP and DBP (23). Obesity and salt intake are the most modifiable risk factors for high BP (24). The present study demonstrated that the SBP, MBP and DBP of middle-aged offspring are increased compared with those of other age groups, including more aged mice. The present results suggest that MHFD induces obesity and hypertension among maternal 

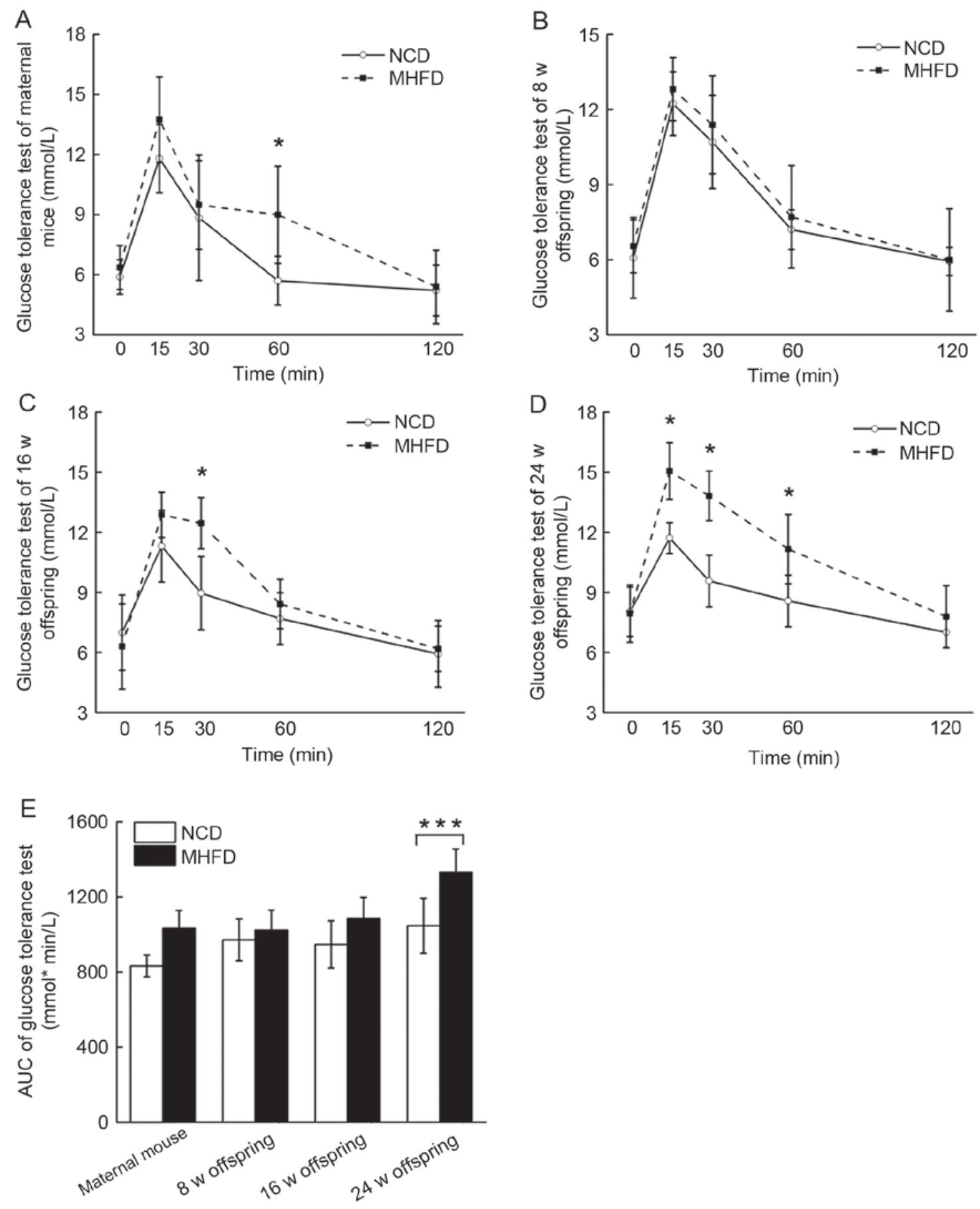

Figure 3. Comparisons of glucose tolerance between NCD and MHFD. (A) Glucose tolerance test of maternal mice. Glucose tolerance tests of offspring at (B) 8 weeks, (C) 16 weeks and (D) 24 weeks, and (E) the AUC of filial glucose tolerance. Values are given as the mean \pm the standard error. "P<0.05 and ${ }^{* * *} \mathrm{P}<0.001$ vs. control. AUC, area under the curve; MHFD, moderate high fat diet; NCD, normal chow diet.

mice and their offspring, and that a MHFD will increase the probability of offspring hypertension by $\leq 25 \%$ if other factors remain unaltered.

Feeding MHFD to WT mice has been demonstrated to cause hyperglycemia and glucose intolerance (10). The oral glucose tolerance test is a method with which to exclude the subsequent manifestation of gestational diabetes mellitus in pregnant women at high risk (25). In the present study, adolescent offspring demonstrate little difference in glucose tolerance. After 16 weeks, a significant increase in glucose tolerance was exhibited. The present results demonstrated that the accumulation of body fat contributes to the formation of insulin resistance in a time-dependent manner. The blood glucose graph of the maternal mice exhibited a similar trend to that described by Murabayashi et al (9).

Compared with the $10-15 \%$ macrophage content observed in lean animals (26), adipose tissue macrophages comprise $45-60 \%$ of stromal cells in obese animals (27). Adipose tissue is the principal site for the long-term storage of nutrients and also regulates systemic metabolism through the release of hormones termed adipokines (28). Obesity increases tissue infiltration by macrophages and polarization to the 
Table II. Comparisons of the wet mass of adipose tissue and major organs between mice fed NCD and mice fed MHFD in offspring of 8,16 and 24 weeks of age.

\begin{tabular}{|c|c|c|c|c|c|c|}
\hline \multirow[b]{3}{*}{ Characteristic } & \multicolumn{6}{|c|}{ Group } \\
\hline & \multicolumn{2}{|c|}{8 weeks } & \multicolumn{2}{|c|}{16 weeks } & \multicolumn{2}{|c|}{24 weeks } \\
\hline & NCD & MHFD & $\mathrm{NCD}$ & MHFD & NCD & MHFD \\
\hline Body weight (g) & $20.012 \pm 2.711$ & $21.703 \pm 2.312$ & $21.315 \pm 1.621$ & $23.213 \pm 1.794$ & $22.720 \pm 2.501$ & $27.401 \pm 3.579$ \\
\hline Kidney (g) & $0.140 \pm 0.021$ & $0.133 \pm 0.051$ & $0.142 \pm 0.020$ & $0.133 \pm 0.015$ & $0.148 \pm 0.030$ & $0.177 \pm 0.034^{\mathrm{a}}$ \\
\hline Subcutaneous fat (g) & $0.097 \pm 0.015$ & $0.147 \pm 0.043^{\mathrm{a}}$ & $0.107 \pm 0.050$ & $0.148 \pm 0.019$ & $0.122 \pm 0.050$ & $0.308 \pm 0.118^{c}$ \\
\hline $\begin{array}{l}\text { Epididymal or } \\
\text { parametrial fat }(\mathrm{g})\end{array}$ & $0.122 \pm 0.04$ & $0.295 \pm 0.252$ & $0.156 \pm 0.051$ & $0.204 \pm 0.061$ & $0.174 \pm 0.071$ & $0.499 \pm 0.190^{c}$ \\
\hline Mesenteric fat (g) & $0.118 \pm 0.058$ & $0.175 \pm 0.041$ & $0.140 \pm 0.044$ & $0.228 \pm 0.037^{\mathrm{c}}$ & $0.150 \pm 0.047$ & $0.344 \pm 0.157^{\mathrm{b}}$ \\
\hline Perirenal fat (g) & $0.022 \pm 0.012$ & $0.067 \pm 0.035^{\mathrm{a}}$ & $0.045 \pm 0.039$ & $0.072 \pm 0.038$ & $0.066 \pm 0.045$ & $0.250 \pm 0.132^{c}$ \\
\hline Spleen (g) & $0.076 \pm 0.011$ & $0.074 \pm 0.011$ & $0.080 \pm 0.022$ & $0.066 \pm 0.014$ & $0.072 \pm 0.010$ & $0.086 \pm 0.028$ \\
\hline Pancreas (g) & $0.088 \pm 0.021$ & $0.108 \pm 0.033$ & $0.124 \pm 0.024$ & $0.111 \pm 0.019$ & $0.116 \pm 0.027$ & $0.119 \pm 0.032$ \\
\hline Liver $(g)$ & $0.878 \pm 0.117$ & $0.979 \pm 0.146$ & $0.963 \pm 0.216$ & $0.977 \pm 0.051$ & $1.092 \pm 0.197$ & $1.077 \pm 0.133$ \\
\hline Heart (g) & $0.149 \pm 0.040$ & $0.135 \pm 0.015$ & $0.141 \pm 0.025$ & $0.127 \pm 0.018$ & $0.142 \pm 0.029$ & $0.144 \pm 0.036$ \\
\hline
\end{tabular}

Values are given as mean \pm standard error. ${ }^{\mathrm{a}} \mathrm{P}<0.05,{ }^{\mathrm{b}} \mathrm{P}<0.01$ and ${ }^{\mathrm{c}} \mathrm{P}<0.001$ vs. NCD mice. MHFD, moderate high fat diet; $\mathrm{NCD}$, normal chow diet.
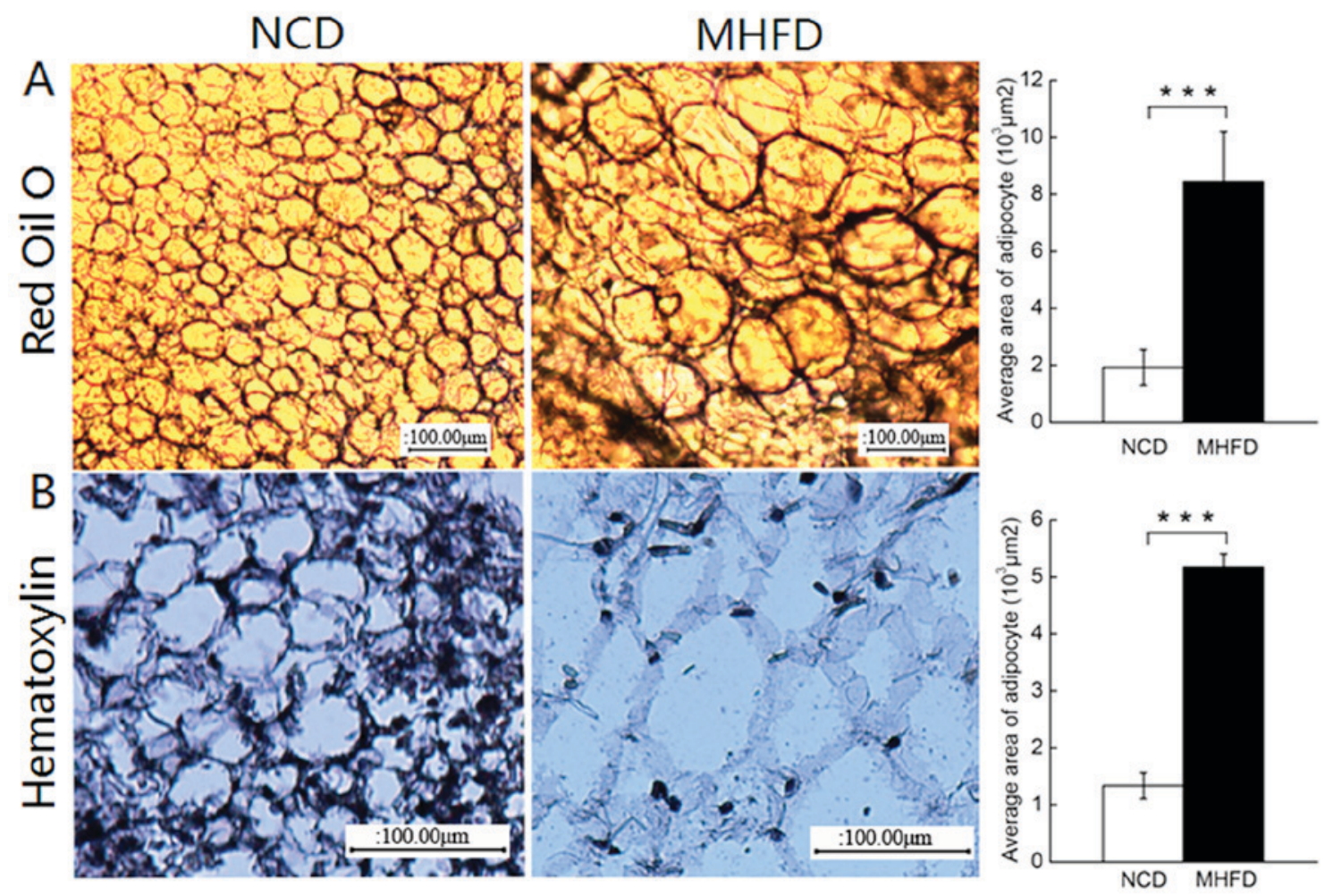

Figure 4. Alterations in adipocyte size between NCD and MHFD offspring. (A) Frozen sections of subcutaneous adipose tissue collected from 24-week old offspring and the average area of adipocytes from frozen sections between NCD and MHFD groups. (B) Paraffin sections of subcutaneous adipose tissue collected from 24-week old mice and the average area of adipocytes from paraffin sections between NCD and MHFD groups. Three horizons were selected at random for each section and 20 cells were measured for each horizon. Values are given as the mean \pm the standard error. ${ }^{* * * *} \mathrm{P}<0.001$. MHFD, moderate high fat diet; NCD, normal chow diet.

pro-inflammatory M1 state (10). TNF- $\alpha$ is an M1 marker cytokine, while IL10 expression is associated with M2 polarization (10). In the present study, the increasing expression of CD68 in the adipose tissue of MHFD offspring demonstrates that obesity elevates tissue macrophage levels. The increased serum TNF- $\alpha$ of MHFD offspring may be due to the increasing proportion of M1 macrophages in adipose tissues; this trophic effect of alternatively activated macrophages is 

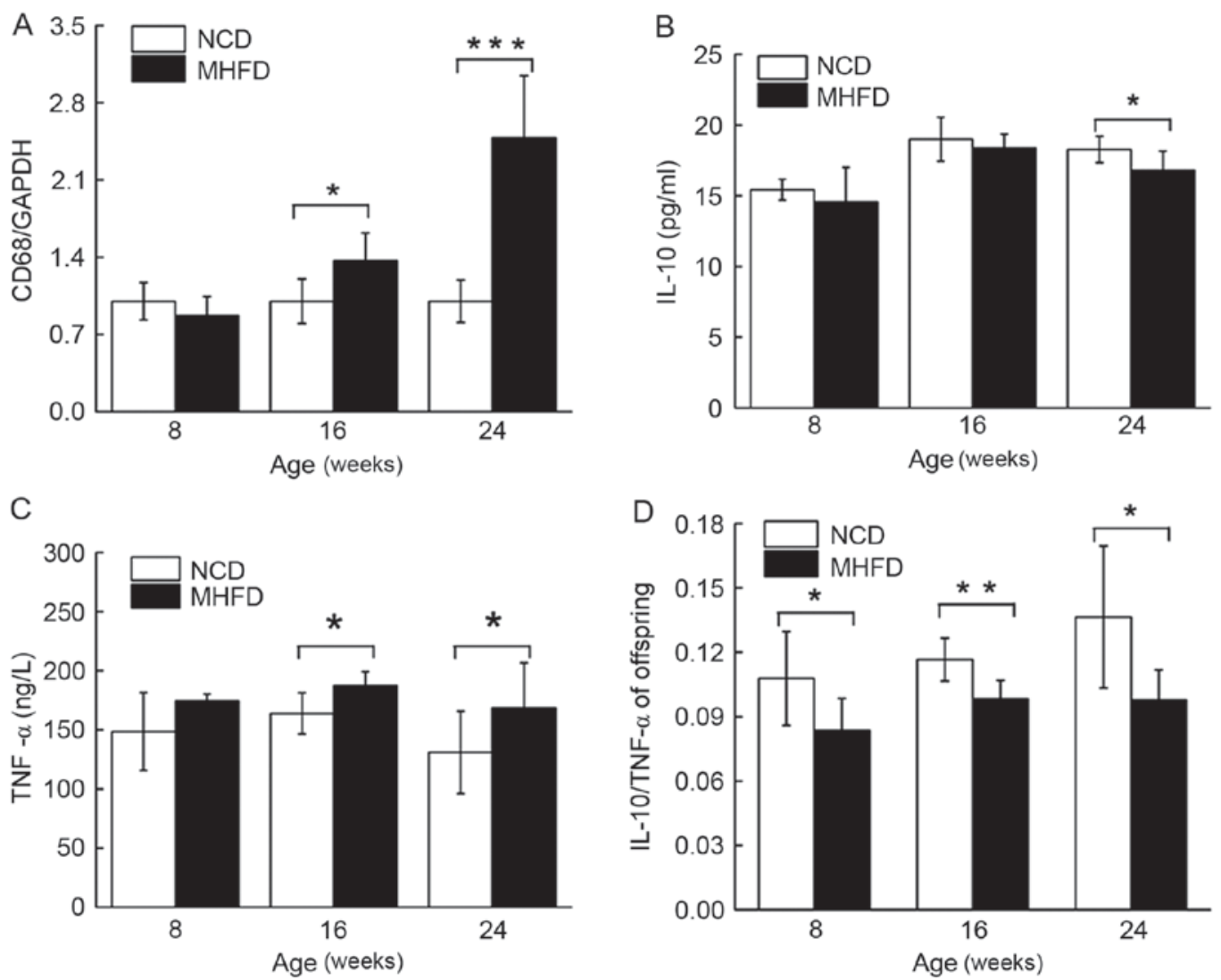

Figure 5. Alterations of inflammatory markers between offspring fed with NCD and those fed with MHFD. (A) The relevant CD68 expression in adipose tissue as demonstrated by reverse transcription-quantitative polymerase chain reaction analysis. (B) The plasma IL-10 concentrations of NCD and MHFD groups in three age groups. (C) Comparisons of the plasma TNF- $\alpha$ concentration in three age groups between NCD and MHFD groups. (D) The plasma IL-10/ TNF- $\alpha$ ratio in three age groups between NCD and MHFD groups. Each experiment was repeated three times. Values are given as the mean \pm the standard error. ${ }^{*} \mathrm{P}<0.05,{ }^{* *} \mathrm{P}<0.01$ and ${ }^{* * *} \mathrm{P}<0.001$. CD68, cluster of differentiation 68; MHFD, moderate high fat diet; NCD, normal chow diet; IL-10, interleukin-10; TNF- $\alpha$, tumor necrosis factor- $\alpha$.

partly mediated by IL-10 secretion, which potentiates insulin action in adipocytes (26). There exists a controversy in previous research around the association between obesity and M2 macrophage content. Following treatment with acute high fat diet, alternative macrophage polarization was promoted in adipose tissue (12). In other studies, the decline of local IL-10 has been reported (10). In the present study, the serum IL-10 protein levels of the young and aged MHFD offspring were significantly decreased compared with those exhibited by NCD offspring, while no significant difference was demonstrated between the middle-aged groups.

Although exercising is among one of the primary treatments for obesity (2), little has been reported about the association between diet-induced obesity and skeletal muscle function. The present study demonstrated that in aged offspring groups, fat accumulation significantly reduced physical endurance capacity by $71 \%$ compared with healthy control mice. Previous research demonstrated that imbalanced local expression of TNF- $\alpha$ and IL-10 leads to inflammation-induced myopathy, including heart failure (14). Therefore, the physical decline of aged offspring may be due, in part, to the long-term imbalance between IL-10 and TNF- $\alpha$.

It was observed that MHFD increased running time by $47 \%$ in the early age MHFD group, compared with the control group. However, the leanness may be the reason for the poor performance of the normal control offspring compared with the
MHFD offspring. Prolonged moderate-level aerobic exercise at $65 \%$ maximum aerobic capacity results in the maximum contribution of fat to the total energy expenditure; at this level, fat may contribute $40-60 \%$ of the total energy expenditure, depending on the duration of the exercise (29). An alteration in body movement and coordination was not demonstrated in the present study. In conclusion, obesity causes metabolic disorders and hypertension, and alterations in physical endurance capacity, one of the underlying reasons for which may be the long-term altered IL-10/TNF- $\alpha$ ratio.

\section{Acknowledgements}

The authors of the present study would like to acknowledge Dr Zhihong Wang of Changchun University of Chinese Medicine (Changchun, China), for the efforts and support by providing the BP2010; Miss Yang Liu of the Biological Experimental Teaching Demonstration Center of Jilin University (Changchun, China), for assistance and help with mouse breeding; and Dr Liming Hao of the Department of Histology and Embryology, College of Life Science, Jilin University Changchun, China), for the suggestions about histology. The present study was supported by the Natural Science Fund Program of China (grant nos. 31070309 and 81200454), Jilin Provincial Bureau of Science and Technology (grant no. 20140307014NY) and Project 985 of Jilin University. 


\section{References}

1. Haslam DW and James WP: Obesity. Lancet 366: 1197-1209, 2005.

2. Lee-Young RS, Ayala JE, Fueger PT, Mayes WH, Kang L and Wasserman DH: Obesity impairs skeletal muscle AMPK signaling during exercise: Role of AMPK alpha 2 in the regulation of exercise capacity in vivo. Int J Obesity (Lond) 35 982-989, 2011.

3. Church TS: Why obesity should be treated as a disease. Curr Sports Med Rep 13: 205-206, 2014.

4. Vahratian A: Prevalence of overweight and obesity among women of childbearing age: Results from the 2002 national survey of family growth. Matern Child Health J 13: 268-273, 2009.

5. Shi P, Yang W, Yu Q, Zhao Q, Li C, Ma X, Jin L, Han X, Zhang Y and Yan W: Overweight, gestational weight gain and elevated fasting plasma glucose and their association with macrosomia in chinese pregnant women. Matern Child Health J 18: 10-15, 2014

6. Hales CN and Barker DJP: Type 2 (non-insulin-dependent) diabetes mellitus: The thrifty phenotype hypothesis. Diabetologia 35: 595-601, 1992.

7. Barker DJ: Maternal nutrition, fetal nutrition, and disease in later life. Nutrition 13: 807-813, 1997.

8. Vogt MC, Paeger L, Hess S, Steculorum SM, Awazawa M, Hampel B, Neupert S, Nicholls HT, Mauer J, Hausen AC, et al: Neonatal insulin action impairs hypothalamic neurocircuit formation in response to maternal high-fat feeding. Cell 156 495-509, 2014

9. Murabayashi N, Sugiyama T, Zhang L, Kamimoto Y, Umekawa T, Ma N and Sagawa N: Maternal high-fat diets cause insulin resistance through inflammatory changes in fetal adipose tissue. Eur J Obstet Gynecol Reprod Biol 169: 39-44, 2013.

10. Han MS, Jung DY, Morel C, Lakhani SA, Kim JK, Flavell RA and Davis RJ: JNK expression by macrophages promotes obesity-induced insulin resistance and inflammation. Science 339 218-222, 2013

11. Di Gregorio GB, Yao-Borengasser A, Rasouli N, Varma V, Lu T, Miles LM, Ranganathan G, Peterson CA, McGehee RE and Kern PA: Expression of CD68 and macrophage chemoattractant protein-1 genes in human adipose and muscle tissues: Association with cytokine expression, insulin resistance, and reduction by pioglitazone. Diabetes 54: 2305-2313, 2005.

12. Ji Y, Sun S, Xia S, Yang L, Li X and Qi L: Short term high fat diet challenge promotes alternative macrophage polarization in adipose tissue via natural killer T cells and interleukin-4. J Biol Chem 287: 24378-24386, 2012.

13. Zhang LY, Sugiyama T, Murabayashi N, Umekawa T, Ma N, Kamimoto Y, Ogawa Y and Sagawa N: The inflammatory changes of adipose tissue in late pregnant mice. J Mol Endocrinol 47: $157-165,2011$.

14. Shreeram S, Ramesh S, Puthan JK, Balakrishnan G, Subramanian R, Reddy MT and Pereira SL: Age associated decline in the conversion of leucine to $\beta$-hydroxy- $\beta$-methylbutyrate in rats. Exp Gerontol 80: 6-11, 2016.
15. Batista ML Jr, Rosa JC, Lopes RD, Lira FS, Martins E Jr, Yamashita AS, Brum PC, Lancha AH Jr, Lopes AC and Seelaender M: Exercise training changes IL-10/TNF-alpha ratio in the skeletal muscle of post-MI rats. Cytokine 49: 102-108, 2010.

16. Basic VT, Jacobsen A, Sirsjö A and Abdel-Halim SM: TNF stimulation induces VHL overexpression and impairs angiogenic potential in skeletal muscle myocytes. Int J Mol Med 34: 228-236, 2014.

17. Kim DS, Cha HN, Jo HJ, Song IH, Baek SH, Dan JM, Kim YW, Kim JY, Lee IK, Seo JS and Park SY: TLR2 deficiency attenuates skeletal muscle atrophy in mice. Biochem Biophys Res Commun 459: 534-540, 2015.

18. Jung SY, Kim DY, Yune TY, Shin DH, Baek SB and Kim CJ: Treadmill exercise reduces spinal cord injury-induced apoptosis by activating the PI3K/Akt pathway in rats. Exp Ther Med 7: 587-593, 2014.

19. Stover KR, Campbell MA, Van Winssen CM and Brown RE: Analysis of motor function in 6-month-old male and female 3xTg-AD mice. Behav Brain Res 281: 16-23, 2015.

20. Xu L and Liu Y: Administration of telmisartan reduced systolic blood pressure and oxidative stress probably through the activation of PI3K/Akt/eNOS pathway and NO release in spontaneously hypertensive rats. Physiol Res 62: 351-359, 2013.

21. Livak KJ and Schmittgen TD: Analysis of relative gene expression data using real-time quantitative PCR and the 2(-Delta Delta C(T)) method. Methods 25: 402-408, 2001.

22. Preston Campbell J, Mulcrone P, Masood SK, Karolak M, Merkel A, Hebron K, Zijlstra A, Sterling J and Elefteriou F: TRIzol and Alu qPCR-based quantification of metastatic seeding within the skeleton. Sci Rep 5: 12635, 2015.

23. Grundy SM: Obesity, metabolic syndrome, and cardiovascular disease. J Clin Endocr Metab 89: 2595-2600, 2004.

24. Ozturk C, Aparci M, Karaduman M, Balta S, Çelik T and Iyisoy A: Relationship of systolic blood pressure and body mass index with left ventricular mass and mass index in adolescents. Angiology 67: 58-65, 2016.

25. Correia-Costa L, Cosme D, Nogueira-Silva L, Morato M, Sousa T, Moura C, Mota C, Guerra A, Albino-Teixeira A, Areias JC, et al: Gender and obesity modify the impact of salt intake on blood pressure in children. Pediatr Nephrol 31: 279-88, 2016.

26. Bitó T, Nyári T, Kovács L and Pál A: Oral glucose tolerance testing at gestational weeks $\leq 16$ could predict or exclude subsequent gestational diabetes mellitus during the current pregnancy in high risk group. Eur J Obstet Gynecol Reprod Biol 121: 51-55, 2005.

27. Lumeng CN, Bodzin JL and Saltiel AR: Obesity induces a phenotypic switch in adipose tissue macrophage polarization. J Clin Invest 117: 175-184, 2007.

28. Weisberg SP, McCann D, Desai M, Rosenbaum M, Leibel RL and Ferrante AW: Obesity is associated with macrophage accumulation in adipose tissue. J Clin Invest 112: 1796-1808, 2003.

29. Wynn TA, Chawla A and Pollard JW: Macrophage biology in development, homeostasis and disease. Nature 496: 445-455, 2013. 